\title{
Diacronie
}

Studi di Storia Contemporanea

$N^{\circ} 18,2 \mid 2014$

Le esposizioni: propaganda e costruzione identitaria

\section{Raviver le «roman national» lusitanien. L'exemple de l'Exposition du Monde portugais de 1940}

\section{Cécile Gonçalves}

\section{(2) OpenEdition}

\section{Journals}

\section{Édition électronique}

URL : http://journals.openedition.org/diacronie/1460

DOI : 10.4000/diacronie.1460

ISSN : 2038-0925

Éditeur

Association culturelle Diacronie

\section{Référence électronique}

Cécile Gonçalves, « Raviver le «roman national» lusitanien. L'exemple de l'Exposition du Monde portugais de $1940 »$, Diacronie [En ligne], N 18, 2 | 2014, document 10, mis en ligne le 01 juin 2014, consulté le 20 avril 2019. URL : http://journals.openedition.org/diacronie/1460 ; DOI : 10.4000/ diacronie. 1460 


\section{Diacronie}

10/

\section{Raviver le «roman national» lusitanien. L'exemple de l'Exposition du Monde portugais de 1940}

Cécile GONÇALVES *

Véritable ode à la "Révolution nationale » menée par Salazar, l’Exposition du Monde portugais de 1940 a été inaugurée en grande pompe alors que l'Europe était ravagée par la guerre. L'Exposition s'inscrivait dans la construction d'un "roman national » souhaitant unifier la nation par une instrumentalisation nationaliste de l'histoire afin d'asseoir la domination du pouvoir salazariste non pas tant sur les populations colonisées que sur les autochtones de la métropole.

\section{Une Exposition grandiose en période de guerre}

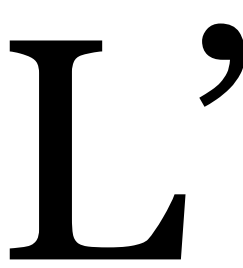

État Nouveau - Estado Novo - salazariste, instauré en 1933 dans le sillage du coup d'État militaire du 28 mai 1926, a placé au cœur de son discours idéologique la «vocation impériale» du Portugal. Depuis l'adoption de l'Acte colonial en 1930, il s'est efforcé de faire «aimer l'Empire» aux Portugais, de susciter des vocations de colons tout en propageant une véritable mystique impériale au sein de la population métropolitaine. La «vocation impériale» du pays constituait, en effet, un thème susceptible de faire consensus parmi la population portugaise, capable de rassembler partisans et opposants de la dictature salazariste. Depuis l'avènement de l'Estado Novo, de nombreuses publications, savantes ou à destination du grand public, aimaient, en effet, à rappeler l'oeuvre « civilisationnelle » des Portugais outre-mer. Ainsi, l'Estado Novo s'est efforcé d'être représenté dans toutes les grandes manifestations coloniales 
internationales de l'époque pour signifier à la face du monde qu'il n'est pas «un petit pays». Le dictateur-président du Conseil Salazar, malgré son programme d'austérité, a accordé d'importants moyens pour que le pays disposât de quatre pavillons imposants lors de l'Exposition coloniale internationale et des pays d'outre-mer de Vincennes en 1931, lors de l'Exposition universelle de Paris en 1937 ou celle de New York en 1939. Il a également veillé à coordonner plusieurs évènements sur son territoire national pour « conscientiser » les Portugais de leur patrimoine colonial. En 1934, la ville de Porto accueillait, pour la première fois, une grande exposition coloniale. Dans le sillage de cette manifestation et, bien que la guerre dévastait l'Europe, le Portugal salazariste, resté neutre, a organisé entre le 23 juin et le 2 décembre 1940 une grande « Exposition du Monde portugais » à Belém sur les bords du Tage, là d'où jadis partaient les caravelles, pour célébrer en grandes pompes les huit cents ans de la fondation du Portugal par Dom Afonso Henriques (1140), les trois cents ans de la Restauration de l'Indépendance (1640) et l'oeuvre « régénératrice » entreprise par Salazar. Symbole de la période de stabilité de la dictature, António Ferro déclarait à ce propos que:

1140 explique 1640, comme 1640 prépare 1940. Ce sont les trois années sacrées de notre histoire, l'année de la naissance, l'année de la renaissance et l'année qui glorifie la résurrection ! Ce que nous allons fêter, ce n'est pas tant le Portugal d'hier, celui de D. Afonso Henriques et de D. João IV, que le Portugal d'aujourd'hui, celui de Carmona et de Salazar ${ }^{1}$.

Au sein de la présidence du Conseil des ministres a été créée une Commission pour procéder à l'organisation des festivités du «Double centenaire de la Nationalité » (Fondation de la Nationalité en 1140 et Restauration de 1640). Le 11 avril et le 2 juin 1938, tandis que la Guerre civile ravageait l'Espagne, deux ordonnances ont été publiées pour nommer les personnalités chargées de constituer la Commission exécutive. Le 28 octobre de la même année, suite à la promulgation du décret-loi $\mathrm{n}^{\circ} 29$ : 087, la Commission nationale des Centenaires a été constituée et ses compétences définies. Júlio Dantas fut nommé par Salazar président de la commission exécutive des commémorations. Augusto de Castro a été nommé commissaire général de l'exposition. Il fut assisté dans sa fonction par Sá e Melo (commissaire adjoint). Le directeur du SPN, António Ferro, en tant que secrétaire général, fut chargé de superviser l'ensemble.

1 FERRO, António cité par LEONARD, Yves, «Le Portugal et ses "sentinelles de pierre". L'exposition du monde portugais en 1940", in Vingtième Siècle. Revue d'histoire. 62, 4/1999. p. 29. 
Malgré un contexte international difficile, l'Europe se dirigeant à grands pas vers le conflit armé, cherchant à s'ancrer dans la tradition des expositions universelles, les commémorations du « Double centenaire » et principalement l'exposition du monde portugais devaient étaler sous les yeux du pays, davantage que sous ceux des peuples voisins en guerre, un résumé de la richesse artistique, commerciale et industrielle du Portugal et de son Empire. Elles traduisaient clairement, en mobilisant des moyens matériels et humains impressionnants, la «portugalité ». Donnant à voir le projet culturel conservateur et passéiste du régime, il s'agissait d'encenser le "génie » portugais. La « race» lusitanienne à qui avait échue la vocation de «montrer des nouveaux mondes au monde » pour paraphraser le poète Camões, de civiliser des contrées sauvages, d'évangéliser de nouvelles peuplades. Nonobstant les vicissitudes du conflit mondial, l'exposition du « monde portugais » se devait d'être monumental afin d'inscrire le régime de Salazar, l'Estado Novo, dans la continuité de l'histoire portugaise tout en signifiant son triomphe sur la République à travers l'instauration d'un État autoritaire, corporatif et chrétien. Le but affiché de l'exposition du « monde portugais » était de faire contraste face au reste du monde en promouvant l'image d'un Portugal impérial à l'apogée de sa puissance, l’image d'un havre de paix aux confins d'une Europe dévastée.

L'exposition a ainsi été inaugurée peu après la défaite française, le 23 juin 1940 en présence du chef de l’État, le Maréchal Carmona, accompagné du président du Conseil Oliveira Salazar, du Patriarche de Lisbonne D. Manuel Cerejeira, du ministre des Travaux publics et maire de Lisbonne, Duarte Pacheco. « Le régime de Salazar [...], en marge du conflit de son temps, commençait un voyage singulier à l’intérieur de son histoire, de son passé2 ${ }^{»}$ souligne l'historien Ramos do Ó. L'exposition du «monde portugais » de 1940 est pourtant restée l'une des dates et l'un des lieux de mémoire de l'Estado Novo. Après sa clôture en décembre, la grande fête de Belém resta dans la mémoire nationale comme l'apogée de la dictature, spectacle provisoire qui devint musée puis référence obligatoire pour des générations. L’historien Yves Léonard écrit :

Par les moyens qu'elle met en œuvre, l'intense mobilisation qu'elle provoque - au point d'apparaître rétrospectivement comme "l'apogée" du régime salazariste -, cette monumentale mise en scène du "monde portugais" s'incarnant dans un vaste empire colonial centralisé autour de Lisbonne, conforte l'image, patiemment dessinée par la dictature, d'un Portugal fort de "huit siècles d'histoire", partagé

${ }^{2}$ Ó, Jorge Ramos do, «Modernidade e tradição: Algumas reflexões em torno da Exposição do Mundo Português», in O Estado Novo : Das origens ao fim da autarcia (1926-1959), [actes], vol. 2, Lisbonne, Fragmentos, 1987, pp. 177-185, p. 177. 
entre tradition et modernité, puissance internationale pacifique, retranchée derrière ses colonies. L’image irréelle d’un Portugal protégé par ses "sentinelles de pierre"3.

\section{Un plan de rénovation patrimoniale et urbaine de la «capitale d'Empire»}

Prévue pour durer six mois, l'Exposition du «Monde portugais» a constitué la clé de voûte du programme officiel des commémorations des «Centenaires». À travers elle, c'est alors l'essence même du projet culturel salazariste qui s'expose dans toute sa splendeur. Elle permet de saisir la manière dont l'Estado Novo s'est efforcé de relier les traits fondamentaux de son nationalisme - autoritarisme, élitisme, paternalisme, conservatisme - à un passé mythique qui est devenu la caution légitimatrice du présent. En moins de deux ans, Lisbonne a changé de visage pour parvenir à éblouir le monde malgré les affres liés à la guerre et à l'occupation nazie d'une grande partie du continent européen.

Les commémorations ont été le prétexte pour lancer un vaste plan de rénovation urbaine et patrimoniale. Plusieurs pièces du patrimoine national ont, en effet, été restaurées parmi lesquelles de nombreux châteaux de l'époque médiévale et une grande partie des collections de tableaux de l'école des primitifs portugais (1450-1550) ${ }^{4}$. On a réouvert le Théâtre de São Carlos, principale salle d'opéra de la capitale lusitanienne, en mai 1940 après avoir effectué une série de travaux d'élargissement de la scène. La première représentation fut celle des Bailados do Verde Gaio, la compagnie nationale de danses folkloriques créée à l'initiative d'António Ferro directeur du Secrétariat de la propagande nationale (SPN) dans l'optique de promouvoir l'authenticité de la culture populaire portugaise. Sur le plan universitaire, peu de temps avant l'ouverture de l'exposition, un Congrès du «Monde portugais » a rassemblé près de 231 historiens portugais et 121 historiens étrangers qui ont présenté pas moins de 515 communications 5 .

\footnotetext{
3 LEONARD, Yves, «Le Portugal et ses "sentinelles de pierre". L'exposition du monde portugais en 1940", in Vingtième Siècle. Revue d'histoire. 62, 2/1999. pp. 27-37.

4 Voir par exemple le catalogue de l'exposition «Cent ans de Primitifs portugais», URL:

< http://legrandatelier.canalblog.com/archives/2011/10/21/22430344.html > [Consulté le 28 mars 2014].

5 Chiffres cités par Júlia Leitão de Barros, «Exposição do Mundo Português», in ROSAS, Fernando, BRANDÃO de BRITO, José Maria (dir.), Dicionário de História do Estado Novo, Venda Nova, Bertrand Editora, 1996, p. 326.
} 
Cette instrumentalisation du passé à des fins nationalistes s'est également incarnée dans la pierre grâce à une politique de travaux publics à grande échelle. Ce qui sera par la suite qualifié péjorativement de «style portugais suave » allait connaître ses heures de gloire. Le résultat de ce courant initié par l'architecte Raul Lino mais propagé par Cottinelli Telmo à la fin des années 1930, fut l'élaboration d'un style imposant, monumental où le modernisme initial fut progressivement évincé par l'esprit nationaliste et on assista à un mélange de style moderniste mâtiné d'Art déco associé à des éléments ornementaux tirés de l'art manuélin et l'époque classique des XVII et XVIIIe siècles. On tentait par la sorte de réaliser une synthèse entre la modernité et la tradition. « À la fois commémoration et reconstitution historique, l'exposition oscille entre tonalité fascisante, avec colonnes imposantes et structures massives - celle prônée par le SPN pour les grandes parades du régime - et l'approche qu'affectionne Salazar, celle du "pays-village" rural et passéiste ${ }^{~}$. Ainsi, en 1940, le Stade national, conçu par Jacobetty Rosa, a été inauguré ainsi que la « Fontaine Lumineuse » - Fonte Luminosa - de l'avenue Afonso Henriques. On a élargi le Musée national d'Art antique et, sous la direction de Luís Cristino da Silva, l'Assemblée nationale a été remodelée selon les canons esthétiques du régime. Dès 1938, une politique volontariste de travaux publics permet de désenclaver la «capitale de l'Empire ». Pour ce faire, les autorités publiques firent bâtir la gare portuaire d'Alcântara - Estação Marítima de Alcântara , la gare fluviale de Belém, l'aéroport a Portela et son avenue, la première autoroute du pays et le viaduc - futur Viaduto Duarte Pacheco -, et la réhabilitation des quartiers de logements sociaux d'Alvalade et du Rostelo situés dans des zones insalubres. Pour améliorer la zone, Salazar avait également décidé que les installations de la Fábrica do Gás - Usine du Gaz - autour de la Tour de Belém seraient démantelées pour désencombrer la place de l'Empire - l'une des plus grandes d'Europe - et donner plus de visibilité aux prochaines installations de l'exposition. Par conséquent, on a démoli de nombreux bâtiments, exproprié des terrains, déplaçé des résidents.

La «grande exposition du Monde portugais» constituait une œuvre pharaonique et coûteuse et, s'il s'est bien agi d'adresser une ode au "glorieux passé » de la nation portugaise, il n'était pourtant pas question pour le dictateur-président du Conseil de déséquilibrer les comptes du pays qu'il venait tout juste de redresser. L'austérité était une politique économique érigée en art de vivre sous l'Estado Novo salazariste à laquelle nul ne pouvait déroger même pour servir la grandeur du Portugal et de son

\footnotetext{
${ }^{6}$ LEONARD, Yves, «Le Portugal et ses "sentinelles de pierre". L'exposition du monde portugais en 1940", op. cit.
} 
Empire. Il fut alors décidé de jouer sur l'illusion puisque tous les édifices allaient être construits dans des matériaux bon marché comme le plâtre, le stuc, le papier ou le bois.

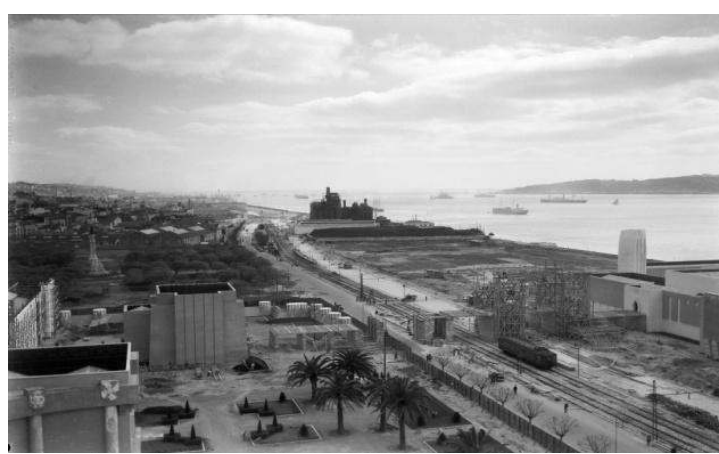

Fig. 1. Vue d'ensemble des travaux de la zone de Belém en vue de l'exposition du Monde portugais (1939)7.

Pendant les quatorze mois que durèrent les travaux, près de 5.00o ouvriers, 15 ingénieurs, 1.000 plâtriers, des centaines de peintres, sculpteurs et décorateurs s'activèrent ${ }^{8}$. La réalisation du plan de l'exposition fut confiée à Cottinelli Telmo (architecte en chef) qui constitua pour ce faire une équipe composée de 12 architectes, 20 sculpteurs et plus de 40 peintres. Toutefois, compte tenu des matériaux employés, l'exposition du «Monde portugais» ne pouvait être que temporaire, non reproductive. Seuls le Monument des Découvertes - Padrão dos Descobrimentos - (reconstruit en pierre calcaire et béton armé d'après l'original en bois en 1960 lors des commémorations des 500 ans de la disparition de l'Infant D. Henrique, le navigateur) et le bâtiment abritant l'actuel musée d'Art populaire (ancien pavillon de la «Vie populaire») ont été conservés.

\section{Une vue d'ensemble du « monde portugais » selon les idéaux du régime}

Prolongement de la «politique de l'Esprit» lancée dès 1933 par António Ferro qui assujettissait la vie culturelle aux idéaux conservateurs, catholiques et ruralistes du régime, l'exposition du «monde portugais» comprenait plusieurs pavillons thématiques s'ancrant dans le «roman national» ou histoire officielle que proposait la

7 Source Archives municipales de Lisbonne, URL: < http://arquivomunicipal.cmlisboa.pt/sala/online/ui/SearchBasic.aspx?filter=AF > [consulté le 28 mars 2014]. De nombreuses photos libres de droit de l'Exposition peuvent être également vues sur ce blog, URL: $<$ http://restosdecoleccao.blogspot.fr/2012/o6/exposicao-do-mundo-portugues-em-1940.html $>$ [consulté le 28 mars 2014].

8 Chiffres cités par LEITÃO de BARROS, Júlia, «Exposição do Mundo Português», in ROSAS, Fernando, BRANDÃO de BRITO, José Maria, Dicionário de História do Estado Novo, Venda Nova, Bertrand Editora, 1996, p. 326. 
weltanschauung réactionnaire du salazarisme. Ce «récit» prend appui à la fois sur ce que l'historien israélien Shlomo Sand appelle une «mytho-histoire des origines » et une " mise en perspective téléologique » pour reprendre une expression de Susanne Citron. L'écriture de l'histoire relève ici ouvertement de la fiction, de l'épopée, de la mythologie afin de «nationaliser » les Portugais. La mise en scène du passé collectif, la théâtralisation de l'histoire, occupe une place fondamentale dans l'exposition du «Monde portugais» de 1940. On entend, dès lors, résonner la formule de Lucien Febvre : «Une histoire qui sert est une histoire serve ». Les autorités publiques ont, en effet, cherché à produire et imposer des représentations et souvenirs communs à l'ensemble des membres de la société portugaise dans le but de légitimer le nouveau pouvoir tout en étant guidé par des préoccupations d'expiation des «péchés » de la République avec l'imposition d'un «ordre moral».

Par conséquent, pour parvenir à rassembler tous les Portugais autour d'une histoire commune et ce, malgré l'austérité imposée par le dictateur président du Conseil depuis avril 1928, le budget de l'évènement fut assez conséquent et atteignait les 35000 ooo d'escudos. L'exposition qui prit la forme d'un immense spectacle populaire, fut une véritable ville dans la ville puisqu'elle s'étendait sur une surface de $560000 \mathrm{~m}^{2}$ dans la partie occidentale de Lisbonne, entre de la Tour de Belém et le Monastère des Jerónimos. Elle reçut, entre le 23 juin et le 2 décembre, environs 3 millions de visiteurs, « un chiffre invérifiable, mais qui, tel quel, soutient honorablement la comparaison face aux 6 à 8 millions de visiteurs estimés par les organisateurs de l'Exposition coloniale internationale de Paris » note, dans son article, Nadia Vargaftig9.

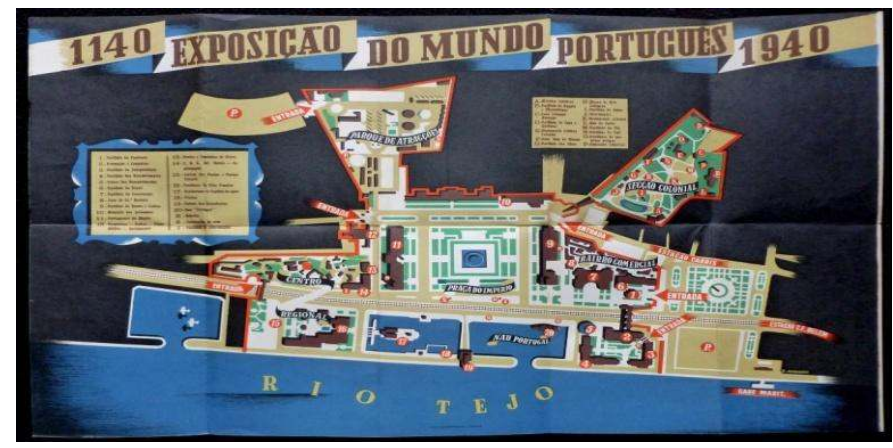

Fig. 2. Plan de l'Exposition du « Monde portugais »10.

\footnotetext{
9 VARGAFTIG, Nadia, «Les expositions coloniales sous Salazar et Mussolini (1930-1940)», Vingtième Siècle. Revue d'histoire, 108, 4/2010, pp. 39-52.

${ }^{10}$ Guide officiel de l'Exposition. Source WIKIPEDIA, «Exposição do Mundo Português», URL:

< http://pt.wikipedia.org/wiki/Exposi\%C3\%A7\%C3\%A3o_do_Mundo_Portugu\%C3\%Aas > [consulté le 28 mars 2014]. Voir également : URL: < http://restosdecoleccao.blogspot.fr/2012/o6/exposicao-do-mundo-portugues-em-1940.html > [consulté le 28 mars 2014].
} 
L'Exposition se divisait en trois grands secteurs : la section historique, la section d'ethnographie coloniale et la section d'ethnographie métropolitaine (appelée centre régional) dans l'objectif de donner une vision d'ensemble de «la civilisation portugaise ». Par sa configuration, l'Exposition du Monde portugais s’inscrivait dans la tradition des Expositions universelles du XIXe siècle vouées à promouvoir la puissance des nations européennes. Non exclusivement consacrée aux colonies, comme a pu être l'Exposition coloniale de Porto en 1934, elle souhaitait montrer la diversité du peuple portugais par-delà les mers et océans. Elle devait « instruire » le peuple portugais selon les idéaux du régime de manière ludique, dans une ambiance bon enfant.

Sans effort, comme dans des dioramas, le visiteur pourrait glisser d'une région à l'autre, d'une colonie à l'autre, passer du Minho au Cap Vert, du Mozambique à Macao pour revenir en Algarve en traversant l'Angola. Il irait d'un palais de Goa à la rue d'un village portugais typique. L'exposition souhaitait donner aux Portugais la sensation de se promener à l'intérieur d'un Portugal qui ne se limitait pas aux seules frontières de la métropole. Le visiteur pouvait découvrir chacune des possessions portugaises au travers de pavillons s'inspirant d'architectures lusitaniennes ou «indigènes ». L'Exposition de Lisbonne de 1940 fut conçue comme un spectacle d'art où la beauté et la couleur des architectures l'emportaient bien souvent sur le strict réalisme. Plusieurs pavillons dits de « style local » furent de libres interprétations, non des reconstitutions fidèles. On eut soin cependant de présenter aux amateurs des arts populaires et « primitifs » des objets authentiques. Comme lors de l'exposition de Porto en 1934 mais avec de moyens plus conséquents, furent donnés des spectacles authentiques comme des processions rituelles ou cérémonie religieuse. On recréa même avec des figurants « natifs » l'arrivée des Portugais dans les différentes contrées africaines et on fit défiler dans leurs costumes et uniformes d'apparat les différentes populations venues de toutes les provinces du «Portugal continental et son Empire » transportées dans la capitale pour l'occasion. Le public fut convié à entendre des orchestres des colonies ou des différentes régions portugaises, ou à admirer des ballets de danses folkloriques traditionnelles comme les paulistes de Trás-os-Montes.

Le monastère des Jerónimos, « joyau de l'art manuélin et symbole de l'âge d'or du début du 16e siècle ", nous dit Yves Léonard ${ }^{11}$, formait la toile de fond de toutes ces manifestations. La Praça do Império en constituait le centre et sur laquelle se faisaient face deux imposants pavillons : celui des «Portugais dans le monde » à l'ouest signé Cottinelli et celui de « l'Honneur et de la ville de Lisbonne » à l'est de Cristino Silva. La

${ }^{11}$ LEONARD, Yves, op. cit. 
Fonte luminosa - la Fontaine lumineuse - située au cœur de la place brillait de mille feux et renforçait l'aspect monumental, démesuré des constructions.

Figg. 3, 4. Vue de la Place de l'Empire d'ouest en est. Au centre, la fontaine lumineuse, à droite le monastère des Jerónimos, devant la pavillon des «Portugais dans le Monde» ${ }^{2}$.
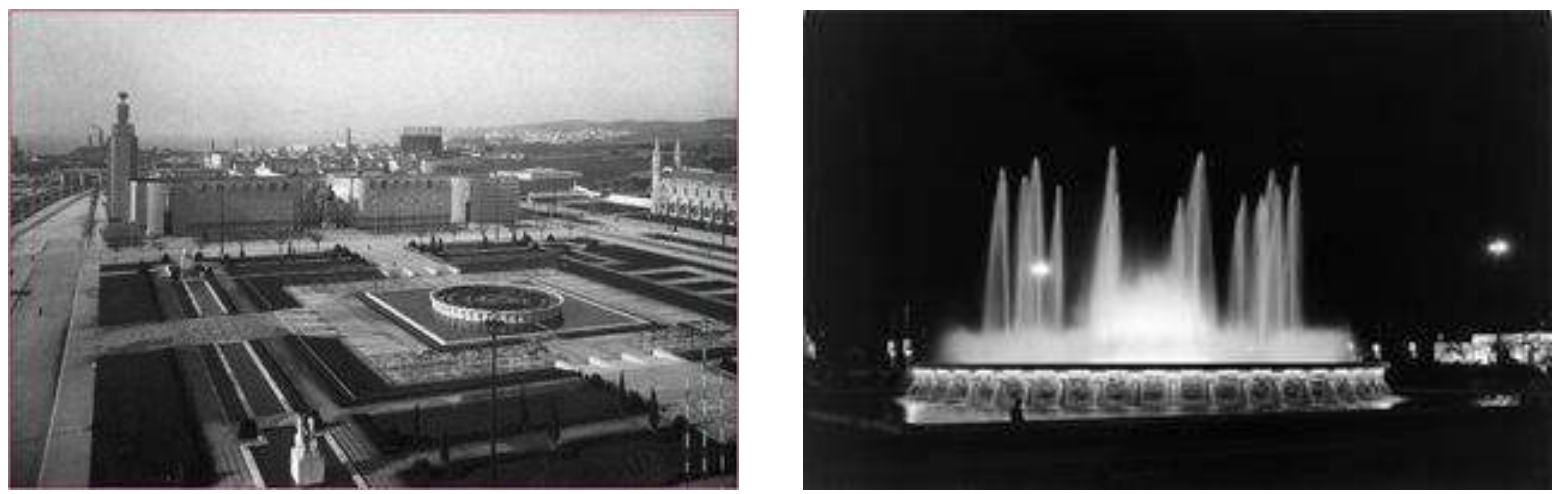

Viennent ensuite les autres pavillons. D'abord, ceux consacrés à l'histoire du Portugal avec le Pavillon de la Fondation, formation et conquête; le Pavillon de l'Indépendance ; le Pavillon des Grandes Découvertes - Descobrimentos - ; le Pavillon de la Colonisation; le Pavillon du Portugal en 1940 dédié à la gloire de la dictature salazariste (élaboré par le SPN d'António Ferro) ; puis, le Pavillon de l'Ethnographie métropolitaine, dont la conception revint également au SPN, fleurant le folklore, les traditions et coutumes locales et disposant d'un Centre régional composé de répliques de villages portugais «typiques » et d'un Pavillon sur la «Vie populaire ». Enfin, la Section d'ethnographie coloniale située dans le «Jardin tropical » imaginée par Henrique Galvão qui avait déjà réalisée l'exposition coloniale de Porto en 1934 et le Pavillon du Brésil. Une reproduction d'un navire amiral semblable à celui que Vasco de Gama avait utilisé pour voguer vers les Indes en 1497 bâptisée « Nau Portugal » était mouillé dans le port derrière le Padrão dos Descobrimentos. Ce monument représentait l'Infant D. Henrique se tenant sur la proue d'une caravelle menant un groupe de grandes figures de l'histoire de l'expansion portugaise montrant ainsi peu de considération envers les incohérences chronologiques. On peut citer parmi les personnalités représentées Camões, Zurara, Pedro Nunes, Gil Eanes, Vasco de Gama, Pedro Álvares Cabral, Diogo Cão, Pêro da Covilhã, Magellan ou encore Afonso de

${ }_{12}$ Source Archives municipales de Lisbonne [en ligne], URL: < http://arquivomunicipal.cmlisboa.pt/sala/online/ui/SearchBasic.aspx?filter=AF > [consulté le 28 mars 2014]. Voir également, URL: < http://restosdecoleccao.blogspot.fr/2012/o6/exposicao-do-mundoportugues-em-1940.html > [consulté le 28 mars 2014]. 
Albuquerque (officier héros des campagnes de "pacification» du Mozambique des années 1895-1898).

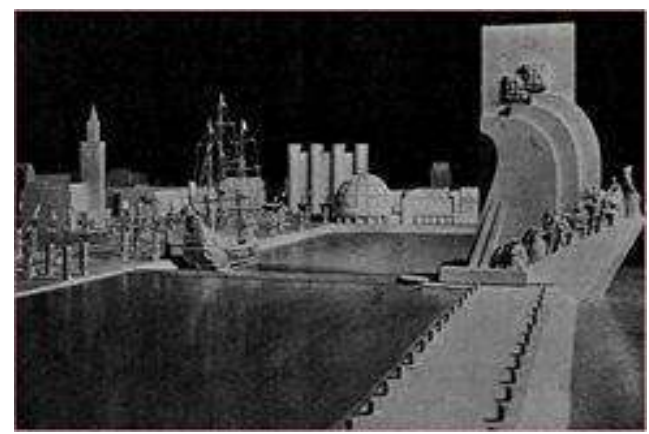

Fig. 5. Vue du Padrão dos Descobrimentos et du Nau de Portugal ${ }^{13}$.

D’immenses colosses figurant des guerriers du Moyen-Âge ornait la «Porte de la Fondation» ${ }^{14}$ et ouvrait sur la section dédiée à l'histoire nationale.

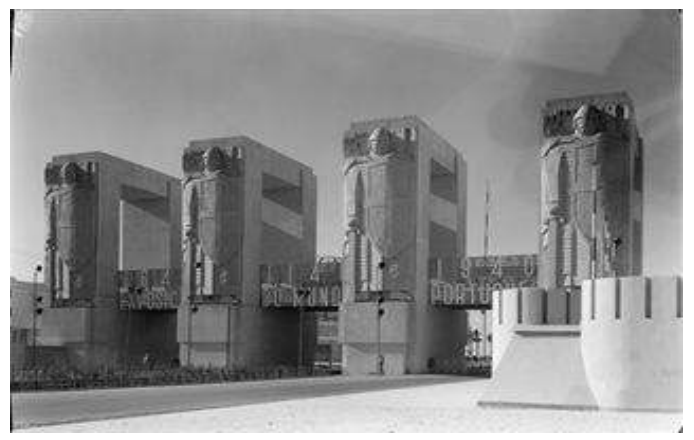

Fig. 6. Vue de la «Porte de la Fondation».

Après avoir traversé le Bairro Comercial e Industrial - le quartier commercial et industriel - vantant les mérites de l'Estado Novo en 1940, de son «Chef» Salazar et sa politique économique, on arrivait au monastère et entrait, ensuite, dans la section coloniale. À l'opposé, après un premier parc d'attractions pour les enfants, en longeant le fleuve on parvenait à la section vouée à l'ethnographie nationale avec son «Centre régional» et son pavillon consacré à «la Vie populaire».

Dans cet appareil complexe, qui s'organise autour de la figure messianique de Salazar, la section coloniale de l'Exposition du monde portugais n'occupe qu'une superficie modeste ; mais elle constitue une des grandes attractions de l'exposition, grâce à la présence des nombreux natifs chargés d'assurer la "représentation

\footnotetext{
${ }^{13}$ Source Archives municipales de Lisbonne, URL:

< http://arquivomunicipal.cm-lisboa.pt/sala/online/ui/SearchBasic.aspx?filter=AF > [consulté le 28 mars 2014].

14 Ibidem.
} 
ethnographique", qui fait partie des attractions traditionnelles des expositions coloniales. Le passé colonial du Portugal est traité et encensé dans l'ensemble de l'exposition: en effet, plusieurs pavillons sont consacrés à l'histoire de la colonisation et l'expansion portugaises dans le monde. La section coloniale, [...], subit [...], la concurrence du Centre régional de l'Exposition, dans un face à face symbolique. [...] L'histoire coloniale du Portugal, représentée par les pavillons de l'expansion et de la colonisation, constitue une des matrices de la nation portugaise, tandis que la vie coloniale contemporaine, reproduite dans la section coloniale, occupe une surface limitée à l'échelle du site. C'est l'histoire coloniale, plus que les réalités coloniales contemporaines, qui constitue, à l'Exposition du monde portugais, le cœur de l’identité impériale portugaise ${ }^{15}$.

Dans la section consacrée à l'ethnographie métropolitaine dit Centro regional, le visiteur pouvait parcourir deux secteurs : une aire d'exposition appelée la «Vie populaire» où l'on faisait l'apologie de l'artisanat traditionnel et le Recinto das Aldeias Portuguesas - espace des villages portugais où des habitations typiques de chaque région du Portugal métropolitain de Trás-os-Montes jusqu'aux Açores et Madère avaient été reconstituées. Dans cet espace, le visiteur pouvait admirer des répliques de maisons «rustiques» emblématiques des différentes régions du pays, alignées le long des rues en présence d' «autochtones» venus de chaque province qui y reconstituaient des scènes du travail quotidien et assuraient des animations. Plusieurs artisans travaillaient dans leurs ateliers, on pouvait assister à des spectacles de danses et de chants folkloriques qui constituaient des attractions très prisées du grand public, on pouvait admirer des intérieurs typiques, il y avait même une taverne et un marché où était vendu des produits du terroir. La section de la «vie populaire» répartie sur plusieurs salles, s'organisait autour de thématiques concernant les costumes, les métiers de l'artisanat comme l'orfèvrerie ou le tissage par exemple, la pâtisserie ou la gastronomie traditionnelles, et exposait diverses charrettes et autres attelages du povo - «menu peuple». Cette section faisait l'éloge de la «culture populaire» et dressait le portrait d'un peuple travailleur, agricole, docile, chrétien, «pauvre mais digne» respectueux de ses traditions, de son histoire et modes de vie séculaires qui vivait d'artisanat et de pêche. Le misérabilisme se voyait esthétisé et érigé en marqueur identitaire de la nation lusitanienne.

Il en allait de même pour la section coloniale où l'«habitat traditionnel» des indigènes avait aussi été reconstitué au cœur de la flore luxuriante du jardin tropical

15 VARGAFTIG, Nadia, op. cit. 
censé apporter une touche d'exotisme. Dans chaque section - qu'elle soit consacrée à la métropole ou aux colonies - le même schéma était décliné: des habitants donnaient vie aux villages reconstitués, des artisans travaillaient sous les yeux du public, d'autres tenaient des stands de souvenirs. Pour les autorités, il s'est agi d'exhiber des hommes et des femmes représentatifs du «monde portugais» pour mieux affirmer le pouvoir de l'Estado Novo, de vanter l'action réformatrice bénéfique du gouvernement salazariste sur ces derniers. Au travers de cette vision idéalisée du monde paysan et colonial, transparaissait dans toute sa splendeur l'idéologie passéiste, ruraliste et impériale du régime.

Enfin, derrière le pavillon de la «Vie populaire», en suivant les bords des quais du Restelo, le visiteur pouvait admirer o Espelho de Água - le miroir d'Eau -, se restaurer au Portugalia ou faire sa digestion en se promenant dans le Jardin des Poètes. Après quoi, il pouvait se recueillir dans la Maison de Santo António, saint patron de Lisbonne, ou s'amuser dans un second parc d'attractions, avant de dépenser ses deniers dans une zone commerciale.

\section{Louange du régime sous forme de plaidoyer passéiste}

L'Exposition du Monde portugais a donc pris l'allure d'un plaidoyer passéiste, la fondation et la conquête de l'Empire étant perçues comme l' «âge d'or» de l'histoire portugaise. «L'époque médiévale - temps de la fondation et de la reconquête sur les infidèles - ainsi que l'épopée des Découvertes et de l'expansion maritime occupent la part belle dans les différents pavillons de l'exposition» affirme Yves Léonard ${ }^{16}$. Grâce à une mise en scène savamment orchestrée, les acteurs publics souhaitaient faire apparaître tout au long de l'exposition «l'âme du Portugal» en exaltant ce qui était considéré comme des grandeurs nationales et gommant les pages sombres de l'histoire nationale. On n'hésita pas à construire des filiations idéologiques arbitraires et à gommer le contexte des évènements et des personnages de l'histoire pour mettre en avant l'idée d'une nation lusitanienne homogène depuis la fondation de 1140. Suivant les consignes officielles de la propagande d'État, les concepteurs de l'exposition tentèrent de restaurer l'unité du pays en orchestrant le retour du «roman national». L'exposition avait pour objectif de rendre palpable la «résurrection nationale» orchestrée par Salazar, de chanter haut et fort les mérites de l'Estado Novo.

${ }^{16}$ LEONARD, Yves, op. cit. 
Le nouveau régime cherchait, en effet, sa légitimité dans une réécriture de l'histoire. Cette exposition martelait le message déjà abondamment diffusé par les services de propagande de l'Estado Novo : le salut temporel et éternel du Portugal et donc la restauration de la communauté nationale. La nécessité pour le gouvernement de se donner une légitimité passait nécessairement par la diffusion d'une image cohérente et unifiée du Portugal et par celle d'une nation contre laquelle rien ne résistait, toujours digne et victorieuse car placée sous la protection de la providence. Ce Portugal de 1940 a son héros. La propagande que l'Exposition du Monde portugais relaye focalisait cette quête de légitimité autour de l'image «messianique» de Salazar, le «sauveur» parvenu à rétablir l'équilibre des finances de la nation, l'artisan de la Constitution de l'Estado Novo adoptée en 1933. Elle l'a situé dans la lignée des grandes figures mythifiées: l'Infant Afonso Henrique, le connétable Nuno Álvares Pereira, les grands navigateurs partis à la découverte et conquête du monde au nom de la chrétienté... Ce message s'articulait autour de la mise en système d'une image rassurante de Salazar, incarnation de la certitude, de la solidité (financière) et de la stabilité (de l'État Nouveau) et par celle de l'héritier héroïque des grandes figures et du sauveur auquel on doit respect, reconnaissance et obéissance. Comme le rappelle Yves Léonard,

\footnotetext{
Les choix auxquels procèdent ces manifestations traduisent une lecture sélective de l'histoire nationale, dominée par un souci d'instrumentaliser celle-ci dans l'optique d'une résurrection nationale. Autrement dit, le libéralisme politique, monarchique aussi bien que républicain, tend à être occulté comme synonyme de décadence pour le Portugal, au profit de l'âge d'or qu'incarneraient les Découvertes et, bien sûr, de la résurrection amorcée le 28 mai 1926 avec l’instauration de la dictature militaire ${ }^{17}$
}

La visite de l'Exposition permettait de retracer l'épopée des Portugais sur les mers, à travers le Pavillon des Découvertes ou le Pavillon du Brésil (ancienne colonie portugaise perdue en 1822). Le Jardin colonial évoquait la vie pittoresque des «indigènes» de l'Afrique portugaise. La figure d'Henri le Navigateur dominait l'ensemble de la propagande des commémorations des Centenaires. La comparaison entre l'Infant et le «mage des Finances» portugaises - Salazar - était l'une des constantes du parcours. Les images du célibataire de Sagres et du «chaste» dictateurprésident du Conseil qui n'a pas d'autre épouse que la nation portugaise, se superposaient. L'un comme l'autre ont dirigé les possessions d'outre-mer sans quitter

${ }_{17}$ LEONARD, Yves, op. cit. 
leur cabinet métropolitain. Et les difficultés économiques du Portugal contemporain étaient assimilées par le Secrétariat de la propagande nationale dirigé par António Ferro, à un nouvel espace de conquête :

\begin{abstract}
Salazar [...] est la personnalité portugaise qui se rapproche le plus, toutes proportions gardées et dans un plan purement psychologique, de l'infant dom Henrique. [...] Comme dom Henrique se penchait à l'école de Sagres, sur les mappemondes et les cartes, aidé par son cosmographe Jaime de Majorque, il se penche, aidé par ses ministres, sur son bureau de la rue de Funchal, sur ce budget qui a déjà été une Mer Ténébreuse et qui s'éclaircit lentement. Les temps ne sont plus les mêmes, les caravelles, aujourd'hui, sont des transatlantiques, des ponts, des écoles, des ports, des usines, des crèches, des cités ouvrières. Et un infant dom Henrique qui ressusciterait à notre époque tourmentée, dans laquelle le bonheur d'un peuple oscille entre ses finances et son économie, pourrait bien être un ministre des Finances, un Oliveira Salazar ${ }^{18}$.
\end{abstract}

Protection et autorité, deux principes qui reflètent le double langage utilisé par le gouvernement de Salazar et qui n'ont cessé d'être répétés tout au long du parcours de l'exposition: un discours consensuel et positif diffusé par la propagande et basé sur la diffusion de valeurs morales s'incarnant dans la supposée «mission civilisatrice» portugaise susceptible de rassembler l'ensemble de la population portugaise en omettant délibérément d'évoquer la violence dont savait faire preuve le régime et sa police politique (répression anticommuniste, l'ouverture du « camp de la mort lente » Tarrefal...). Cette exposition a d'abord été conçue pour canaliser l'adhésion des Portugais vers la personne du président du Conseil et implicitement vers le gouvernement, entretenir et amplifier l'admiration que doit susciter le chef de l'État mais surtout le président du Conseil des ministres que le discours et l'imagerie plaçaient en-dehors des luttes de pouvoir et des contingences matérielles. Ainsi, ce «culte du sauveur» était-il intimement lié à l'évocation d'un passé idéalisé d'où ont été exclues toutes divisions et contradictions. Aussi la mythification des grandes figures s'accompagnait-elle de l'exaltation de la terre, de la ruralité, du monde paysan, de l'artisanat, du régionalisme, de la mère et de la famille.

L'Exposition du monde portugais allait essentiellement se borner à être une œuvre d'«éducation nationale» selon les fameuses «leçons de Salazar»: des affiches éditées en 1938 et distribuées dans toutes les écoles primaires du pays. Celles-ci visaient à

${ }^{18}$ FERRO, António, Salazar, le Portugal et son Chef, Paris, Grasset, 1934, pp. 261-262 
transmettre les valeurs ardemment défendues par l'État Nouveau salazariste telles que l'incontestable supériorité d'un «État fort», autoritaire, sur les régimes libéraux; l'action efficace et bénéfique du gouvernement et de son «chef» Salazar; le rôle subalterne de la femme cantonnée à n'être qu'une épouse et une mère; la charité (chrétienne); le catéchisme (catholique); la glorieuse histoire de la nation et de son Empire qui fait que «le Portugal n'est pas un petit pays».
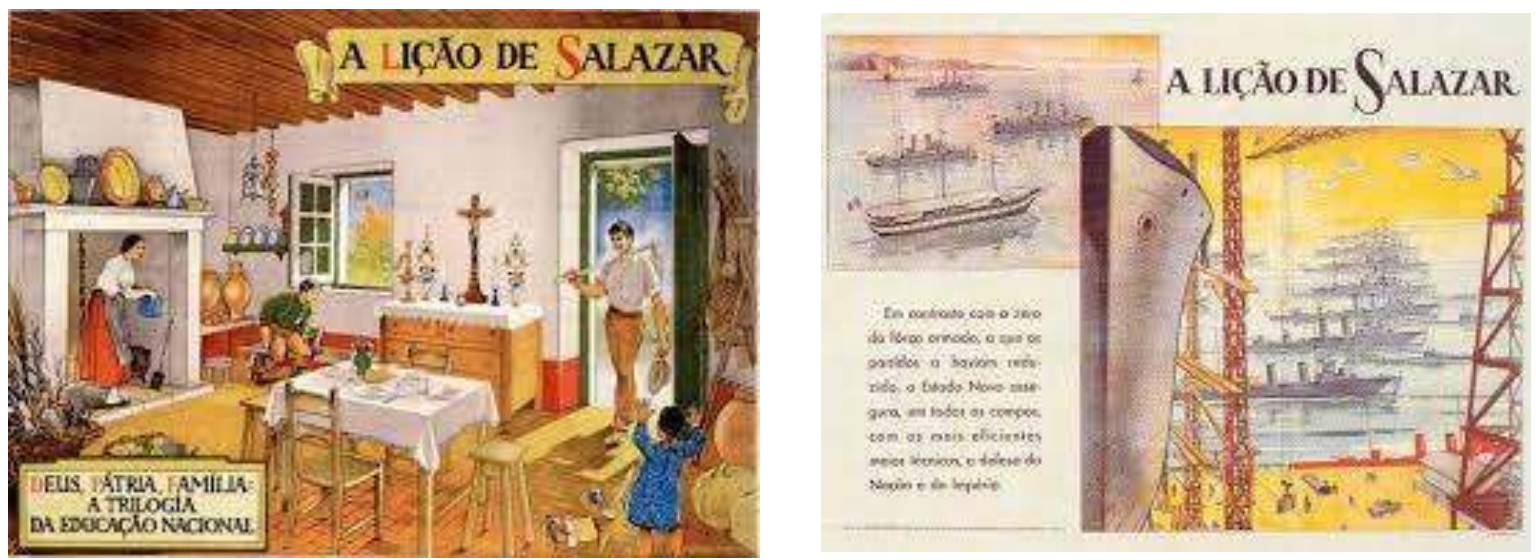

Figg. 7, 8: «Dieu, Patrie et Famille: la trilogie de l'Éducation nationale»; «Défense de la Nation et de l'Empire»19.

Lors de l'exposition de 1940, s'il s'est agi de faire «aimer» l'Empire, il a surtout été question de vulgariser à l'usage du peuple portugais les piètres slogans de la propagande salazariste : la mise en valeur des colonies, l'Empire, remède miracle à la crise, «la terre qui ne ment pas » (pour reprendre une expression du maréchal Pétain), la famille, la patrie. L'exposition était supposée révéler l'existence d'un «génie national». Dès lors, l'utilisation massive de ces thématiques à travers des mises en scène de la vie quotidienne participait de cette conception, réactualisée au XIXe siècle et liée à l'affirmation des nationalismes, qui définit la nation comme un organisme autonome, régi selon ses propres lois, évoluant et se transformant de l'intérieur, en dehors de toute influence extérieure. Cette patrie, celle des ancêtres et des morts, cette mère-terre nourricière et féconde du travail des Portugais, ce foyer qui réunit les forces vives à l'image du foyer familial, puiserait dans son seul corps ses forces et sa jeunesse. Contre la décadence des temps modernes issus de la Révolution française de 1789 et parvenue jusqu'au Portugal par l'instauration de la Monarchie constitutionnelle en 1822 puis de la République en 1910, il s'agissait de restaurer les valeurs d'ordre, de hiérarchie, d'honneur et de dévouement à la communauté nationale. Cette quête des

${ }^{19}$ Affiches libres de droit [en ligne], URL: < http://noseahistoria.wordpress.com/2011/12/12/alicao-de-salazar/ > [consulté le 29 mars 2014]. 
origines de la grandeur était explicitement visible dans la plupart des pavillons de l'exposition notamment celui de «l'Honneur et de la ville de Lisbonne».

Le culte de la lignée des ancêtres renvoie à l'idée d'un corps national cohérent, homogène et soudé par une tradition inexpugnable qui implique l'exclusion de l'autre, de l'étranger. La réécriture de l'histoire apparaissait comme intimement liée à l'éloge du domaine colonial portugais, viatique indispensable au relèvement national. Comme sans doute jamais auparavant, l'Empire allait devenir un mythe compensateur, un objet de propagande permanent contribuant à fixer en profondeur la viabilité du régime. Tout au long de la visite des différents pavillons, on allait redire aux Portugais les bienfaits du régime qui oeuvrait à l'amélioration du niveau de vie de tous les Portugais, les bénéfices de l'apostolat colonial pour «la rééducation des peuples arriérés», le loyalisme reconnaissant des populations soumises et les réalisations du régime dans la métropole et les territoires d'outre-mer. Il était question de «donner aux Portugais conscience de leur Empire» et de leur grandeur. Après la fermeture de l'exposition le 2 décembre 1940, les Portugais ne pourraient désormais oublier que la nation se «régénérait» grâce au programme du gouvernement salazariste et qu'ils possédaient un vaste Empire. Celui-ci cesserait d'être une entité vague, un thème à discours pour devenir une réalité tangible. Ni le Portugal continental ni les colonies ne devaient plus jamais être perçus comme des terra incognita.

Par conséquent, bien plus que d'être tournée vers le rayonnement international, l'Exposition s'adressait d'abord aux Portugais, elle devait renforcer leur confiance dans la grandeur de la nation lusitanienne et son Empire tout en affermissant la confiance envers le nouveau régime seul capable de préserver l'héritage multiséculaire de ce pays «vieux de huit siècles ». Bien plus, l'esprit colonial devait pénétrer les masses populaires. Salazar avait ainsi demandé qu'on insistât sur les réalisations de la politique économique qu'il avait mis en œuvre dès son arrivée au ministère des Finances en 1928 et les progrès dus à la promulgation de l'Acte colonial en 1930 et l'institutionnalisation de l'Estado Novo en 1933 par rapport à la «dispendieuse » République. Dès lors, s'expliquent qu'aient été soulignés dans chaque pavillon les moindres réalisations sociales (comme la construction d'écoles ou de voies ferrées) et les progrès de l'hygiène et de la santé publique sur l'ensemble des territoires portugais. Les photographies de réalisations industrielles, les statistiques sur le mouvement commercial, les collections d'échantillons suivaient de près les arts décoratifs, les collections de masques et fétiches, les costumes traditionnels régionaux et autres objets de la « culture populaire» de façon à intéresser le grand public et qu'il puisse faire sien les messages du régime. 
À l'issue des commémorations du «Double centenaire » en décembre 1940, la dictature salazariste apparaît comme renforcée. L'enthousiasme de la population pour l'exposition du « Monde portugais » a contribué à asseoir sa domination sur les esprits. En mettant savamment en scène une vision nationaliste et artificielle de l'histoire lusitanienne, le régime a su se doter d'une légitimité et donner du Portugal l'image d'un «havre de paix» en Europe ainsi que l'illusion d'être une grande puissance impériale sur la scène internationale. L'Estado Novo est ainsi apparu comme l'artisan de la paix et le garant de l'intégrité nationale face à l'adversité. L'exposition de 1940, en propageant le credo du régime par le spectacle et la fête, a donné à voir l'incroyable efficacité de la machine de propagande salazariste et contribué à faire du président du Conseil un homme providentiel, le plaçant alors sur un pied d'égalité avec l'Infant D. Henrique. Tout avait également été mis en œuvre pour que cet évènement puisse passer à la postérité et devenir un «lieu de mémoire». Un film documentaire a été réalisé par António Lopes Ribeiro sur l'exposition du « Monde portugais » afin que son souvenir reste gravé dans la mémoire collective et puisse régulièrement être invoqué pour renforcer l'aura du régime autoritaire. L'exposition du «Monde portugais» constitue le symbole de l'apogée du régime salazariste : un moment d'extrême complicité entre les artistes et l'Estado Novo qui favorisa la fabrication du consensus autour des idéaux défendus par le régime, la marque d'une stabilisation de la dictature tout en étant, paradoxalement, le tournant crucial de la lente agonie du régime. En effet, peu de temps après, en 1945, la chute des forces de l'Axe allait signifier la fin de la période de stabilité et entraîner la première des crises politiques qu'aurait à affronter la dictature pour survivre. 


\section{* L'auteur}

Cécile Gonçalves est doctorante en Etudes politiques à l'Ecole des Hautes Etudes en Sciences Sociales, Paris; rattachée au Centre d'Etudes Sociologiques et Politiques Raymond Aron (CESPRA); doctorante en Histoire contemporaine à l’Université Picardie Jules Vernes, Amiens ; et rattachée au Centre d'Histoire des Sociétés, des Sciences et des Conflits, Faculté d'histoire et de géographie.

URL: < http://www.studistorici.com/progett/autori/\#Gonçalves >

\section{Per citare questo articolo:}

GONÇALVES, Cécile, «Raviver le roman national lusitanien. L'exemple de l'Exposition du Monde portugais de 1940», Diacronie. Studi di Storia Contemporanea : Le esposizioni: propaganda e costruzione identitaria, 29/6/2014,

URL: < http://www.studistorici.com/2014/6/29/Goncalves_numero_18/ >

\section{Diacronie Studi di Storia Contemporanea $\beta$ www.diacronie.it}

Risorsa digitale indipendente a carattere storiografico. Uscita trimestrale. redazione.diacronie@hotmail.it

Comitato di redazione: Jacopo Bassi - Luca Bufarale - Elisa Grandi - Deborah Paci - Fausto Pietrancosta - Matteo Tomasoni - Luca Zuccolo 